Results A two-compartment model best fitted the data. Current weight, post-menstrual age (PMA) and serum creatinine were the significant covariates for clearance (CL). After model validation, simulations showed that a loading dose $(25 \mathrm{mg} / \mathrm{kg})$ and a maintenance dose $(15 \mathrm{mg} / \mathrm{kg}$ twice daily if $<35$ weeks PMA and $15 \mathrm{mg} / \mathrm{kg}$ three times daily if $\geq 35$ weeks PMA) achieved the AUC0-24 target earlier than a standard 'Blue Book' dosage regimen in more than $89 \%$ of the treated patients.

Conclusions The results of a population meta-analysis of vancomycin data have been used to develop a new dosing regimen for neonatal use and assist in the design of the modelbased, multinational European trial, NeoVanc.

Disclosure(s) Nothing to disclose

\section{PROSPECTIVE EVALUATION OF A POPULATION PHARMACOKINETIC MODEL OF PANTOPRAZOLE FOR OBESE CHILDREN}

${ }^{1}$ A Chapron, ${ }^{1,2}$ S Abdel-Rahman, 1,2,3V Shakhnovich*. ${ }^{1}$ Children's Mercy Kansas City; ${ }^{2}$ University of Missouri-Kansas City School of Medicine, Kansas City, MO; ${ }^{3}$ University of Kansas Medical Center, Kansas City, KS, USA

\subsection{6/archdischild-2019-esdppp.27}

Background Pharmacokinetic (PK) data for proton pump inhibitors, acid suppressive medications commonly prescribed to children, are lacking for obese children who are at greatest risk for acid related disease. Our aim was to evaluate the performance of the only published population PK model of pantoprazole for obese children, in an independent cohort of obese and non-obese children.

Methods A published 2-compartment structural model, ${ }^{1}$ modified to exclude transit compartments for delayed absorption, was used to predict the PK of pantoprazole (PAN) oral suspension, immediate release $\left(k_{\mathrm{a}}=7.3 \mathrm{hr}^{-1}\right)$. Calculated population parameters and covariate relationships (e.g., weight, CYP2C19 genotype) were extracted. Predictions were based on dose, sampling times, and covariates from 57 children (617 years; $21 \%$ obese, $28 \%$ overweight) who received a single dose PAN and had plasma PAN concentrations collected at 10 time-points over 8 hours. Model predictive performance was assessed visually and by relative root mean squared error (RMSE), with mean ratio of predicted-to-observed area under the concentration time curve (AUC) compared via one-way ANOVA across weight groups, defined by body mass index for age (10-84th percentile normal-weight, 85-94th percentile overweight, $>95$ th percentile obese; $\alpha=0.05, \mathrm{R})$.

Results The model generally over-predicted observed PAN concentrations (RMSE 194\%). Ratios of predicted versus observed AUC were not significantly different among obese, overweight and normal-weight children (1.5 vs. 1.7 vs. 2.2, $\mathrm{p}=0.06)$; however, a trend toward better model prediction was observed in the subset of obese children.

Conclusion Observed PAN PK deviated from model predictions, which may be due to differences in patient demographics or PAN formulation. A validation study using a delayed release PAN formulation is in progress, with the overarching goal of understanding PAN disposition, and appropriate dose selection, for obese children, who are at potential risk for drug over- or under-dosing using commonly employed dosing strategies in pediatrics (e.g., $\mathrm{mg} / \mathrm{kg}$, weight-tiered).

\section{REFERENCES}

1. Shakhnovich, et al. A Population-Based Pharmacokinetic Model Approach to Pantoprazole Dosing for Obese Children and Adolescents. Paediatr Drugs 2018 Oct;20(5):483-495.

Disclosure(s) Nothing to disclose

\section{O28 VARIABILITY IN LIVER ANATOMY AND PHYSIOLOGY IN CHILDREN PARTICIPATING IN PHARMACOKINETIC STUDIES}

${ }^{1}$ A Friesen, ${ }^{2} \mathrm{~J}$ Radford, ${ }^{3,4} \mathrm{~S}$ Chan, ${ }^{3,4} \mathrm{~N}$ Mardis, ${ }^{1,3,4} \mathrm{~V}$ Shakhnovich* ${ }^{1}$ University of Kansas Medical Center, Kansas City; ${ }^{2}$ Kansas State University, Manhattan, KS; ${ }^{3}$ Children's Mercy Kansas City; ${ }^{4}$ University of Missouri-Kansas City School of Medicine, Kansas City, MO, USA

\subsection{6/archdischild-2019-esdppp.28}

Background Obesity-related changes in liver anatomy and physiology (e.g., hepatic fat infiltration) may be important sources of interindivdual variability in hepatic drug metabolism and relevant covariates for physiologically-based pharmacokinetic (PBPK) models. The aim of this investigation was to quantify variability in hepatic fat fraction (HFF) and hepatic volume in children participating in PK studies, utilizing a novel, non-invasive, magnetic resonance imaging (MRI) sequence. $^{1}$

Methods Children, without a known diagnosis of fatty liver disease, enrolled in a PK study for hepatic CYP2C19 and CYP3A4 substrates, had hepatic volume and total HFF estimated using MRI proton density fat fraction (PDFF) and HFF assessed via conventional MRI spectroscopy (MRSFF) using a region of interest in the right upper hepatic lobe (LiverLab, Siemens Healthcare). Patient anthropometrics, laboratories and LiverLab outcomes were compared between obese and nonobese children, using independent student t-test, and associations explored via Spearman's correlation $(\rho)$; SPSSv24, $\alpha=0.05$. Obesity was defined by body mass index (BMI) $\geq$ 95th percentile for age; clinically significant liver adiposity defined as $\mathrm{HFF}>5 \%$.

Results 25 children (7-20 years; 56\% obese) had evaluable MRI data. Liver volume ranged $911-2227 \mathrm{~cm}^{3}$, MRSFF $1.6-$ $34.8 \%$ and PDFF $2.1-31.1 \%$. Liver volume and HFF significantly correlated with BMI (both $\rho=0.6, \mathrm{p}<0.01$ ), but not age (both $\rho=0.3, \mathrm{p}>0.11)$. Liver volume $(1574.5 \pm 367.1$ vs $1284.8 \pm 216.3, \quad \mathrm{p}=0.04), \quad \mathrm{MRSFF} \quad(8.9 \pm 8.4$ vs $2.8 \pm 1.2$, $\mathrm{p}=0.02)$, PDFF $(8.9 \pm 7.0$ vs $3.4 \pm 1.3, \mathrm{p}=0.07)$ and alanine aminotransferase (ALT; $37.7 \pm 15.8$ vs $26.8 \pm 3.6 \mathrm{IU} / \mathrm{L}, \mathrm{p}=0.02$ ) were higher in obese vs non-obese children. HFF $>5 \%$ and ALT $>40$ were only observed in obese children.

Conclusion Liver volume and adiposity varied substantially among children and may be important covariates for pediatric PBPK models, especially for obese children. HFF $>5 \%$ and ALT $>40$ were only observed in obese children. Recently, $24 \%$ reduction in clearance of azithromycin, a CYP3A4 substrate, was reported for children with ALT $>40 .^{2}$ Our PK analyses are in progress.

\section{REFERENCES}

1. Caussy C, Reeder SB, Sirlin CB, et al. Noninvasive, quantitative assessment of liver fat by MRI-PDFF as as endpoint in NASH trials. Haptology 2018;68(2):76372.

2. Zheng Y, Liu SP, Xu BP, et al. Population Pharmacokinetics and Dosing Optimization of Azithromycin in Children with Community-Acquired Pneumonia. Antimicrob Agents Chemother 2018:62 (9):e00686-18. 